\title{
Hofstadter butterfly of graphene with point defects
}

\author{
Selcen İslamoğlu, M. Ö. Oktel, and Oğuz Gülseren \\ Department of Physics, Bilkent University, 06800 Ankara, Turkey \\ (Received 28 March 2012; revised manuscript received 28 April 2012; published 7 June 2012)
}

\begin{abstract}
We investigate the structure of Hofstadter's butterfly of graphene with point defects under a perpendicular magnetic field. We use a tight-binding method with interactions up to second-nearest neighbors. First of all, we present the Hofstadter butterfly spectrum of pure graphene, including all four valence orbitals with second-order hopping. To model defects, we perform calculations within an enlarged unit cell of seven carbon atoms and one defect atom. We find that impurity atoms with smaller hopping constants result in highly localized states which are decoupled from the rest of the system. The bands associated with these states form a nearly $E=0 \mathrm{eV}$ line. On the other hand, impurity atoms with higher hopping constants are strongly coupled with the neighboring atoms. These states modify the Hofstadter butterfly around the minimum and maximum values of the energy by forming two self-similar bands decoupled from the original butterfly. We also show that the bands and gaps due to the impurity states are robust with respect to the second-order hopping.
\end{abstract}

DOI: 10.1103/PhysRevB.85.235414

PACS number(s): 73.22.Pr, 71.55.-i, 71.70.Di, 73.22.Gk

\section{INTRODUCTION}

The band structure of graphene was first calculated by Wallace in 1947, and he reported the unusual semimetallic behavior of this material. ${ }^{1}$ Graphene became a very popular field of research, especially after the isolation of a single layer by mechanical exfoliation, ${ }^{2,3}$ and its electronic structure has been deeply investigated. ${ }^{4}$ The existence of Dirac points makes the band structure of graphene unique. It is then desirable to understand how this extraordinary band structure will be modified by a strong magnetic field.

The behavior of Bloch electrons under a magnetic field is interesting in general. The pioneering work on (twodimensional) electronic systems was done by Peierls ${ }^{5}$ for the adoption of the magnetic field to the system, and then Hofstadter $^{6}$ used this model in order to investigate the energy spectrum of a square lattice in the magnetic field. This self-similar spectrum was then called the "Hofstadter butterfly." There have been a lot of studies about the Hofstadter butterflies ${ }^{7,8}$ which focus on the artificial lattices rather than the physical ones. For example, the energy spectra for the square, triangular, and honeycomb lattices ${ }^{9}$ were calculated even with the next-nearest-neighbor interactions. ${ }^{10}$ In addition, the Hofstadter spectrum for the hexagonal lattice was reported. ${ }^{11-13}$ There are also several studies on the fractal energy spectrum of bilayer graphene and carbon nanotubes. ${ }^{14-18}$

Although the Hofstadter butterfly for perfect graphene has been well understood, the effect of impurities on this spectrum has been investigated less. As any real sample would contain a concentration of point defects, it is important to systematically study their influence on the electronic structure. The first study of such point defects considered the effect of vacancies on the Landau levels of graphene. ${ }^{19}$ It has been shown that vacancies introduce new states between the Landau levels and the presence of a vacancy lattice introduces extra bands to the spectrum. In this paper, we present a systematic study in which both vacancies and impurities are considered. Furthermore, we investigate the evolution of impurity bands with coupling strength. These bands are a result of the impurity lattice, and their impact on magnetotransport will be studied elsewhere.

The tight-binding methodology works well for the energy spectrum calculation of graphene as a function of magnetic flux. ${ }^{12}$ In the case of Hofstadter-Rammal butterflies, the usual unit cell of graphene with two atoms in the basis is enough to model the fractal energy spectrum. In this study, we investigate the effects of substitutional point defects such as vacancy or impurity on the electronic structure. We treat one of the atoms in the unit cell as a vacancy with zero hopping constants or an impurity atom with different hopping constants from the rest of the lattice. The effect of impurities can be modeled by introducing a dilute impurity lattice, which requires the study of a larger unit cell. In order to study defects with low concentration, in this paper, we use an enlarged unit cell which has eight atoms in the basis. For this case, we obtain an impurity concentration of $1 / 8=12.5 \%$. In such a large unit cell there is no direct hopping between impurities even in the presence of second-order interactions. We also point out that our method is applicable to any general defect concentration.

This paper is organized as follows: First, we outline the adoption of the tight-binding methodology to graphene described within an enlarged unit cell in Sec. II. Then, we label one of the atoms in the enlarged unit cell as an impurity or vacancy. We change the hopping constant(s) of this defect atom and present the resulting butterflies in Sec. III. This section is divided into Sec. III A and Sec. III B, in which only the first-order interaction and both the first- and the second-order interactions are included in the calculations, respectively. Last, we conclude briefly in Sec. IV.

\section{METHODOLOGY}

Since we have eight atoms in the unit cell, as shown in Fig. 1, the tight-binding Hamiltonian is an $8 \times 8$ matrix: 


$$
H=\left[\begin{array}{llllllll}
H^{A A} & H^{A B} & H^{A C} & H^{A D} & H^{A E} & H^{A F} & H^{A G} & H^{A H} \\
H^{B A} & H^{B B} & H^{B C} & H^{B D} & H^{B E} & H^{B F} & H^{B G} & H^{B G} \\
H^{C A} & H^{C B} & H^{C C} & H^{C D} & H^{C E} & H^{C F} & H^{C G} & H^{C H} \\
H^{D A} & H^{D B} & H^{D C} & H^{D D} & H^{D E} & H^{D F} & H^{D G} & H^{D H} \\
H^{E A} & H^{E B} & H^{E C} & H^{E D} & H^{E E} & H^{E F} & H^{E G} & H^{E H} \\
H^{F A} & H^{F B} & H^{F C} & H^{F D} & H^{F E} & H^{F F} & H^{F G} & H^{F H} \\
H^{G A} & H^{G B} & H^{G C} & H^{G D} & H^{G E} & H^{G F} & H^{G G} & H^{G H} \\
H^{H A} & H^{H B} & H^{H C} & H^{H D} & H^{H E} & H^{H F} & H^{H G} & H^{H H}
\end{array}\right] .
$$

Each element of this matrix gives the interaction terms between the atoms with corresponding labels. The elements of the tightbinding matrix are scalar expressions when we consider only the $p_{z}$ orbitals. If we take all the orbitals into account, then each of the elements is a matrix itself rather than a scalar expression. We introduce the magnetic field to the system by the Peierls substitution:

$$
\hbar \mathbf{k} \rightarrow \hbar \mathbf{k}-\frac{e \overrightarrow{\mathbf{A}}}{c}
$$

For convenience, we use Landau gauge to describe the perpendicular magnetic field with a vector potential $\overrightarrow{\mathbf{A}}=(0, B x, 0)$. Since the wave vectors are modified, we have new phase factors due to the magnetic field which are in the line integral form:

$$
e^{i \vartheta_{\text {magnetic }}}=e^{-2 \pi i \frac{e}{\hbar} \int_{\vec{R}_{m_{\beta}, n_{\beta}}}^{\vec{R}_{m_{\alpha}, n_{\alpha}}} \overrightarrow{\mathbf{A}} \cdot \vec{d} l}
$$

where $\vec{R}_{m_{\alpha}, n_{\alpha}}=m_{\alpha} \vec{a}_{1}+n_{\alpha} \vec{a}_{2}$, with $\alpha$ and $\beta$ being the labels of the atoms in the enlarged unit cell, and the integral is to be evaluated through the line connecting the neighboring atoms. We introduce the flux quanta as $\phi_{0}=h / e$ and the amount of flux passing through the enlarged unit cell as $\phi=6 \sqrt{3} B a^{2}$. So the normalized magnetic flux can be expressed as $\phi / \phi_{0}=$ $p / q$, with two coprime integers $p$ and $q$. By rewriting the atomic wave functions in the separable form along the $x$ and $y$ directions, i.e., $\varphi(x, y)=\varphi(x) e^{i k_{y} y}$, we end up with the phase factors in new and neater forms. For instance,

$$
e^{i \vartheta_{B G}}=e^{-i k_{y}\left|\vec{a}_{2}\right|+i \frac{\pi}{2} \frac{\phi}{\phi_{0}}\left(m_{a}+5 / 12\right)} .
$$

All the phase factors due to the first-order interactions are in the same form, except the additive fractions to $m_{a}$ in the parentheses. This occurs due to the conversion of all the other labeled indices to the ones of the atom labeled A. Under these circumstances, we have a new matrix equation which can be called the generalized Harper's equation ${ }^{20}$ involving three matrices,

$$
\Psi_{m}=U_{m} \Psi_{m}+V_{m} \Psi_{m+1}+W_{m} \Psi_{m-1},
$$

where $\Psi_{m}$ is a vector,

$$
\Psi_{m}=\left[\begin{array}{c}
\psi\left(m_{a}\right) \\
\psi\left(m_{b}\right) \\
\psi\left(m_{c}\right) \\
\psi\left(m_{d}\right) \\
\psi\left(m_{e}\right) \\
\psi\left(m_{f}\right) \\
\psi\left(m_{g}\right) \\
\psi\left(m_{h}\right)
\end{array}\right]
$$

and $U_{m}, W_{m}$, and $V_{m}$ are the following matrices when we consider only the first-order interactions and the $p_{z}$ orbitals:

$$
\begin{aligned}
& U_{m}=\left[\begin{array}{cccccccc}
0 & 0 & 0 & 0 & 0 & 0 & H_{\pi} & 0 \\
0 & 0 & 0 & H_{\pi} e^{i \vartheta_{B D}} & 0 & 0 & H_{\pi} e^{i \vartheta_{B G}} & H_{\pi} \\
0 & 0 & 0 & H_{\pi} & 0 & 0 & 0 & 0 \\
0 & H_{\pi} e^{i \vartheta_{D B}} & H_{\pi} & 0 & H_{\pi} e^{i \vartheta_{D E}} & 0 & 0 & 0 \\
0 & 0 & 0 & H_{\pi} e^{i \vartheta_{E D}} & 0 & H_{\pi} & H_{\pi} e^{i \vartheta_{E G}} & 0 \\
0 & 0 & 0 & 0 & H_{\pi} & 0 & 0 & 0 \\
H_{\pi} & H_{\pi} e^{i \vartheta_{G B}} & 0 & 0 & H_{\pi} e^{i \vartheta_{G E}} & 0 & 0 & 0 \\
0 & H_{\pi} & 0 & 0 & 0 & 0 & 0 & 0
\end{array}\right] \\
& W_{m}=\left[\begin{array}{cccccccc}
0 & 0 & 0 & 0 & 0 & H_{\pi} e^{i \vartheta_{A F}} & 0 & H_{\pi} e^{i \vartheta_{A H}} \\
0 & 0 & 0 & 0 & 0 & 0 & 0 & 0 \\
0 & 0 & 0 & 0 & 0 & H_{\pi} e^{i \vartheta_{C F}} & 0 & H_{\pi} e^{i \vartheta_{C H}} \\
0 & 0 & 0 & 0 & 0 & 0 & 0 & 0 \\
0 & 0 & 0 & 0 & 0 & 0 & 0 & 0 \\
0 & 0 & 0 & 0 & 0 & 0 & 0 & 0 \\
0 & 0 & 0 & 0 & 0 & 0 & 0 & 0 \\
0 & 0 & 0 & 0 & 0 & 0 & 0 & 0
\end{array}\right], \quad V_{m}=\left[\begin{array}{cccccccc}
0 & 0 & 0 & 0 & 0 & 0 & 0 & 0 \\
0 & 0 & 0 & 0 & 0 & 0 & 0 & 0 \\
0 & 0 & 0 & 0 & 0 & 0 & 0 & 0 \\
0 & 0 & 0 & 0 & 0 & 0 & 0 & 0 \\
H_{\pi} e^{i \vartheta_{F A}} & 0 & H_{\pi} e^{i \vartheta_{F C}} & 0 & 0 & 0 & 0 & 0 \\
0 & 0 & 0 & 0 & 0 & 0 & 0 & 0 \\
0 & 0 & 0 & 0 & 0 & 0 & 0 & 0 \\
H_{\pi} e^{i \vartheta_{H A}} & 0 & H_{\pi} e^{i \vartheta_{H C}} & 0 & 0 & 0 & 0 & 0
\end{array}\right] .
\end{aligned}
$$


The index $m_{a}$ is periodic in $q$ with a period of $4 q$. Hence, our new Hamiltonian is a $32 q \times 32 q$ matrix with eight atoms in the enlarged unit cell, and each atom is connected to $4 q$ atoms. Equation (1) is a periodic equation where $m$ runs from 1 to $4 q$. However, when $m=1$, we have $\Psi_{0}$, and similarly, when $m=4 q$, we get $\Psi_{4 q+1}$. Since we have the periodic boundary conditions, we have to retain these wave functions within the magnetic unit cell via Bloch's condition:

$$
\psi\left(m_{a}+4 q\right)=e^{i k_{x} a_{1 x} 4 q} \psi\left(m_{a}\right) .
$$

As a result, we have a new matrix called the $A_{m}$ matrix which is our new Hamiltonian:

$$
\left[\begin{array}{c}
\Psi_{1} \\
\Psi_{2} \\
\vdots \\
\Psi_{4 q-1} \\
\Psi_{4 q}
\end{array}\right]=\left[\begin{array}{ccccccc}
U_{1} & V_{1} & 0 & 0 & \cdots & 0 & W_{1}^{*} \\
W_{2} & U_{2} & V_{2} & 0 & 0 & \cdots & 0 \\
0 & W_{3} & U_{3} & V_{3} & 0 & \cdots & 0 \\
\vdots & \vdots & \ddots & \ddots & \ddots & \ldots & \vdots \\
V_{4 q}^{*} & 0 & 0 & \cdots & 0 & W_{4 q} & U_{4 q}
\end{array}\right]\left[\begin{array}{c}
\Psi_{1} \\
\Psi_{2} \\
\vdots \\
\Psi_{4 q-1} \\
\Psi_{4 q}
\end{array}\right] .
$$

The eigenvalues of this $A_{m}$ matrix generate the HofstadterRammal butterfly when plotted as a function of $p / q=\alpha$. A similar procedure can be carried out in order to introduce the second-order interactions. The second-order interactions produce new phase factors and alter the $U_{m}, W_{m}$, and $V_{m}$ matrices by modifying the phase factors for previous elements and generating new nonzero entries. For a more accurate description, the second-order interactions are important even though their values are small. To go one step further, we can also take all the orbitals $\left(s, p_{x}, p_{y}\right.$, and $\left.p_{z}\right)$ into account. This will enlarge the $A_{m}$ matrix to a $128 q \times 128 q$ matrix because there are four orbitals for each of the eight atoms. The resulting butterfly with the second-order interactions is presented in Fig. 2, which includes many bands, so it is more complicated than the one with only $p_{z}$ orbitals. Note that in this study, we use the well-established tight-binding parameter for graphene listed in Table I. This complicated energy spectrum is not transparent enough to study the effects of the impurities. Since the aim of this paper is to understand the effects of the point defects, from now on we just concentrate on the $p_{z}$ orbitals, as in previous studies.

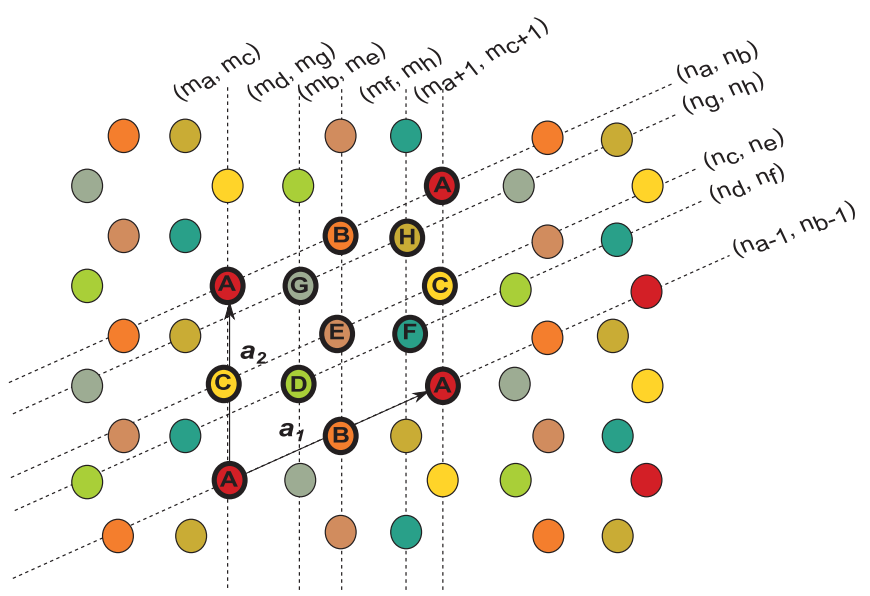

FIG. 1. (Color online) Enlarged unit cell for graphene which is suitable for point defect calculations. The lattice vectors are $\vec{a}_{1}=$ $\hat{x} 3 a+\hat{y} a \sqrt{3}$ and $\vec{a}_{2}=\hat{y} 2 a \sqrt{3}$.

\section{RESULTS AND DISCUSSION}

The energy spectrum for pure graphene was obtained in the previous section. Now, we concentrate on the effects of the impurities by modifying the $A_{m}$ matrix. The Hofstadter butterflies for the range of impurity hopping strengths are displayed in Figs. 3 and 4, which constitute the results of this work.

The pure cases for up to first- and second-nearest-neighbor interactions are displayed in Figs. 3(c) and 4(c). The horizontal axes denote the magnetic flux $\alpha$, which is from 0 to 24 . This difference in the $\alpha$ range occurs for two reasons. First of all, we enlarged our unit cell; as a result, the amount of magnetic flux per unit cell was increased. Second, the second-order interactions bring an extra envelope-like periodicity to the butterflies. In order to visualize the butterflies through one whole period, we have to span more $\alpha=p / q$ when we consider the second-order interactions. For the sake of comparison between the first- and the second-order interactions, we prefer to plot the butterflies of the first-order interactions

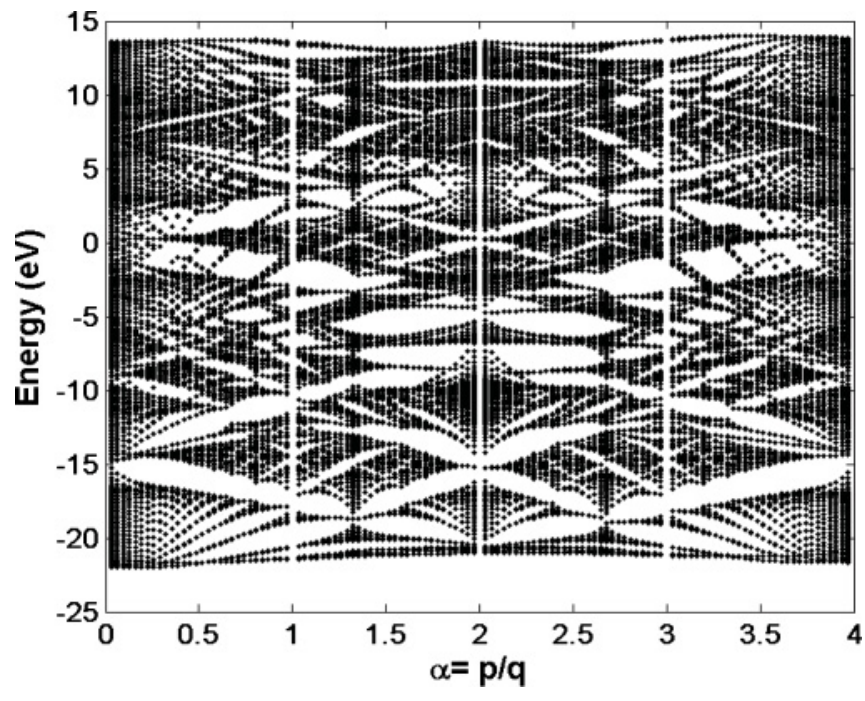

FIG. 2. The Hofstadter butterfly spectrum for graphene with all of the orbitals. Both the first- and the second-nearest-neighbor interactions are included. The tight-binding parameter are displayed in Table I. 
TABLE I. Tight-binding interaction parameters for graphene from Ref. 21. All values are in eV. $\epsilon_{2 s}$ and $\epsilon_{2 p}$ are the self-interactions of the $s$ orbitals and the $p$ orbitals. $H_{s s}$ and $H_{s p}$ are the interactions of the $s$ orbital with the neighboring $s$ orbital and the $s$ orbital with the neighboring $p$ orbital, respectively. $H_{p p \sigma}$ and $H_{p p \pi}$ are the interactions of the $\sigma$ and $\pi$ orbitals with the neighboring $\sigma$ and $\pi$ orbitals.

\begin{tabular}{|c|c|c|c|c|c|}
\hline \multicolumn{2}{|c|}{$\begin{array}{l}\text { On-site } \\
\text { energies }\end{array}$} & \multicolumn{2}{|c|}{$\begin{array}{l}\text { Nearest-neighbor } \\
\text { interaction } \\
\text { parameters }\end{array}$} & \multicolumn{2}{|c|}{$\begin{array}{c}\text { Next-nearest-neighbor } \\
\text { interaction } \\
\text { parameters }\end{array}$} \\
\hline$\epsilon_{2 s}$ & -7.3 & $H_{s s}$ & -4.30 & $H_{s s}^{2}$ & -0.18 \\
\hline \multirow[t]{3}{*}{$\epsilon_{2 p}$} & 0.0 & $H_{s p}$ & 4.98 & $H_{s p}^{2}$ & 0.0 \\
\hline & & $H_{p p \sigma}$ & 6.38 & $H_{p p \sigma}^{2}$ & 0.35 \\
\hline & & $H_{p p \pi}$ & -2.66 & $H_{p p \pi}^{2}$ & -0.10 \\
\hline
\end{tabular}

with the same range of $\alpha$ in accordance with the second-order results.
We created the basis for the calculation of the Hofstadter butterflies for graphene with point defects. By treating one of the eight atoms as a vacancy or an impurity we obtain a concentration of $12.5 \%$, as explained above. We choose the atom labeled $\mathrm{E}$ to be an imperfection. The vacancy case corresponds to the case where atom $\mathrm{E}$ has zero hopping constants. Similarly, by changing the hopping constant(s) involving the interactions among atom $\mathrm{E}$, we can monitor the effects of different substitutional impurities on the electronic structure.

\section{A. First-nearest neighbors}

The Hofstadter butterflies of graphene with point defects can be seen in Fig. 3 for the first-order interactions. As we mentioned before, the pure case is given by Fig. 3(c). This is the usual Hofstadter butterfly for graphene. Since we have only the first-nearest-neighbor interactions, the spectrum is symmetric

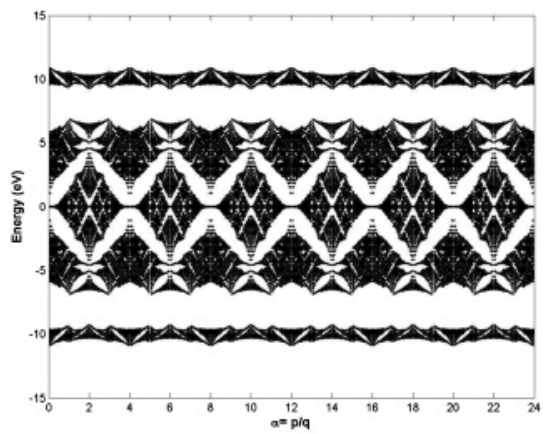

(a)

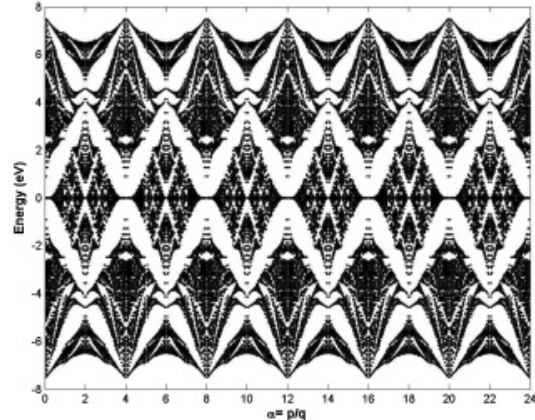

(d)

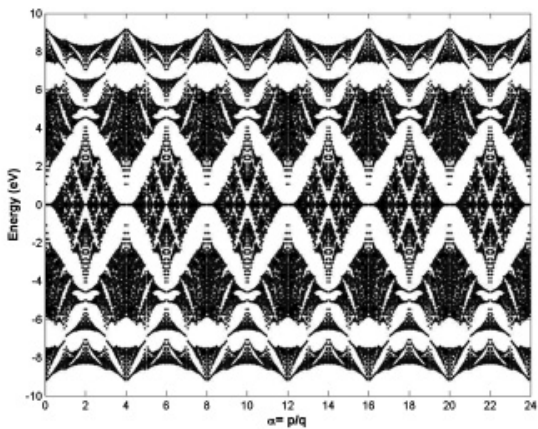

(b)

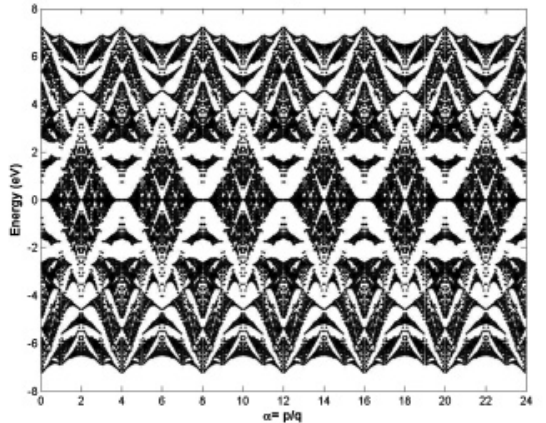

(e)

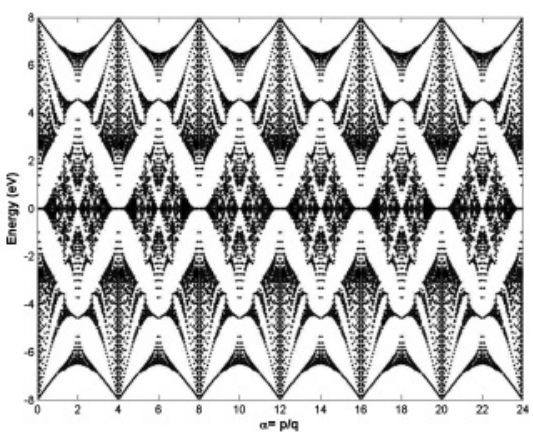

(c)

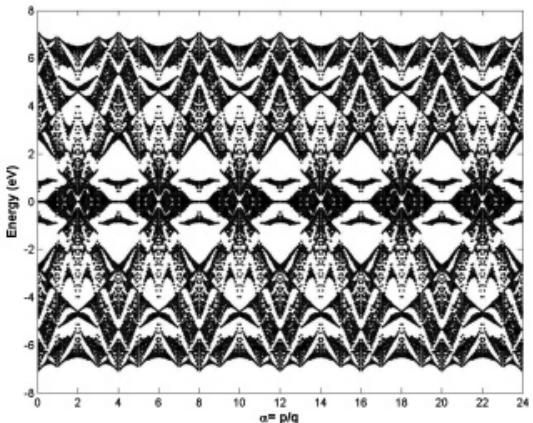

(f)

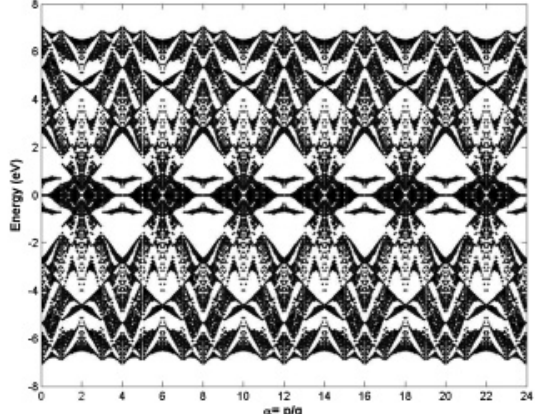

(g)

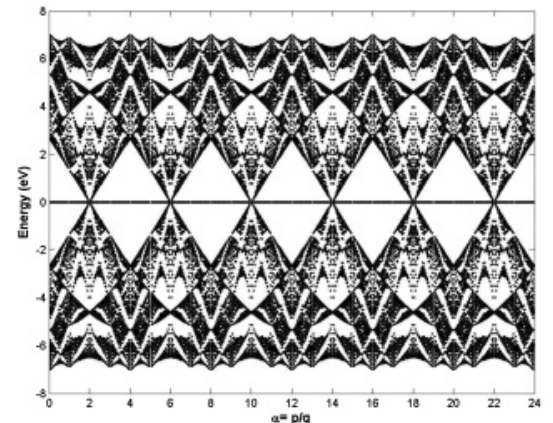

(h)

FIG. 3. The Hofstadter butterfly spectra of graphene with point defects with the first-order interactions. (a) Impurity with larger hopping constant, $H_{p p \pi}^{E}=2 H_{p p \pi}$. (b) Impurity with large hopping constant, $H_{p p \pi}^{E}=\frac{3}{2} H_{p p \pi}$. (c) Pure case where all of the atoms are carbon atoms, $H_{p p \pi}^{E}=H_{p p \pi}$. (d) Impurity with smaller hopping constant, $H_{p p \pi}^{E}=\frac{3}{4} H_{p p \pi}$. (e) Impurity with smaller hopping constant, $H_{p p \pi}^{E}=\frac{1}{2} H_{p p \pi}$. (f) Impurity with smaller hopping constant, $H_{p p \pi}^{E}=\frac{1}{4} H_{p p \pi}$. (g) Impurity with smaller hopping constant, $H_{p p \pi}^{E}=\frac{1}{5} H_{p p \pi}$. (h) Vacancy case where atom $\mathrm{E}$ is missing, $H_{p p \pi}^{E}=0$. 


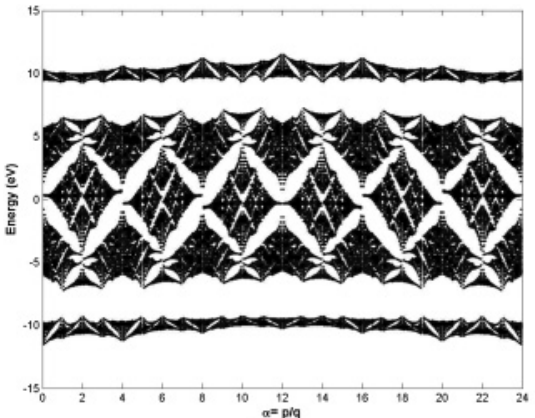

(a)

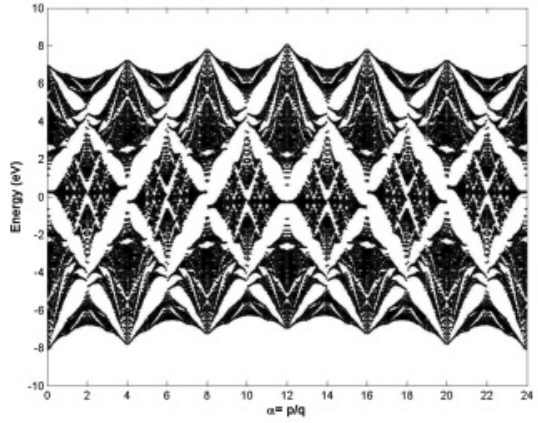

(d)

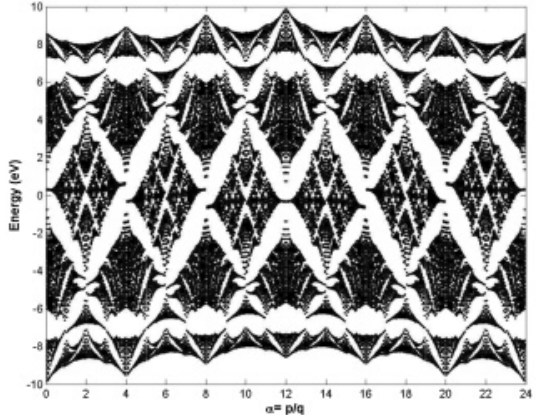

(b)

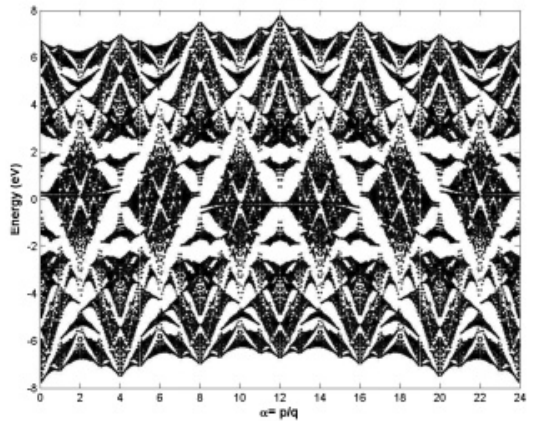

(e)

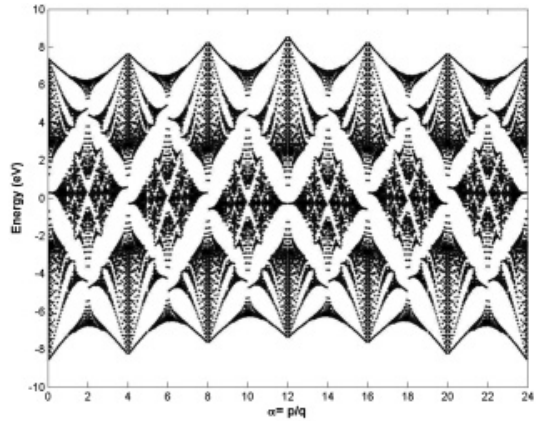

(c)

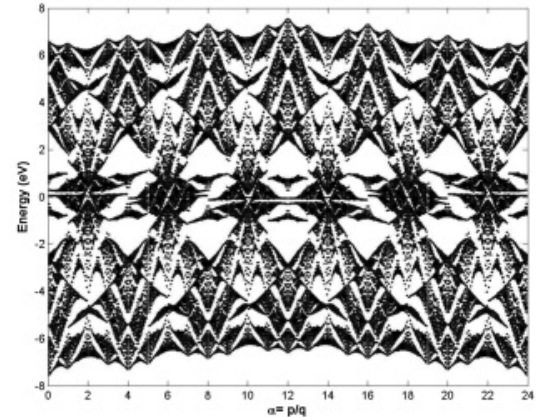

(f)

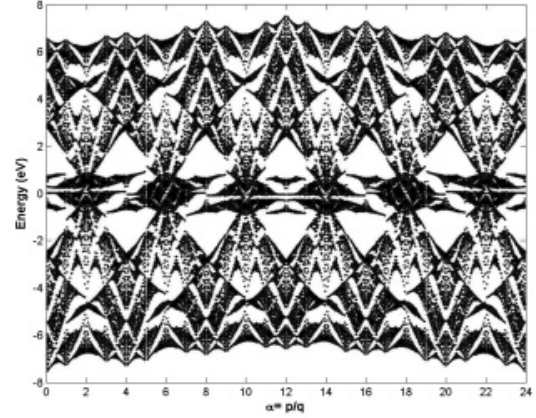

(g)

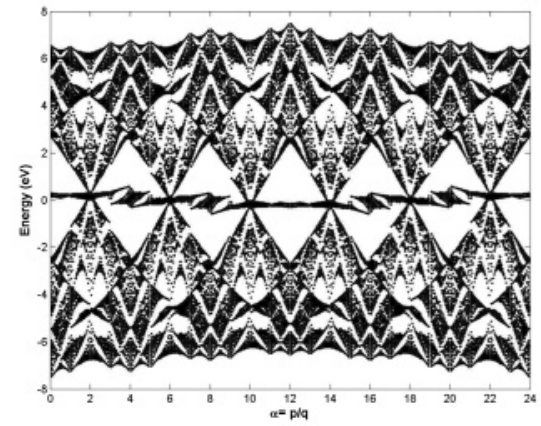

(h)

FIG. 4. The Hofstadter butterfly spectra of graphene with point defects for the first- and second-order interactions. (a) Impurity with larger hopping constants, $H_{p p \pi}^{E}=2 H_{p p \pi}$ and $H_{p p \pi}^{2(E)}=2 H_{p p \pi}^{2}$. (b) Impurity with large hopping constants, $H_{p p \pi}^{E}=\frac{3}{2} H_{p p \pi}$ and $H_{p p \pi}^{2(E)}=\frac{3}{2} H_{p p \pi}^{2}$. (c) Pure case where all of the atoms are carbon atoms, $H_{p p \pi}^{E}=H_{p p \pi}$ and $H_{p p \pi}^{2(E)}=H_{p p \pi}^{2}$. (d) Impurity with smaller hopping constants, $H_{p p \pi}^{E}=\frac{3}{4} H_{p p \pi}$ and $H_{p p \pi}^{2(E)}=\frac{3}{4} H_{p p \pi}^{2}$. (e) Impurity with smaller hopping constants, $H_{p p \pi}^{E}=\frac{1}{2} H_{p p \pi}$ and $H_{p p \pi}^{2(E)}=\frac{1}{2} H_{p p \pi}^{2}$. (f) Impurity with smaller hopping constants, $H_{p p \pi}^{E}=\frac{1}{4} H_{p p \pi}$ and $H_{p p \pi}^{2(E)}=\frac{1}{4} H_{p p \pi}^{2}$. (g) Impurity with smaller hopping constants, $H_{p p \pi}^{E}=\frac{1}{5} H_{p p \pi}$ and $H_{p p \pi}^{2(E)}=\frac{1}{5} H_{p p \pi}^{2}$. (h) Vacancy case where atom E is missing, $H_{p p \pi}^{E}=0$ and $H_{p p \pi}^{2(E)}=0$.

around $E=0 \mathrm{eV}$. When we introduce an impurity to the system with the smaller hopping constant of $H_{p p \pi}^{E}=\frac{3}{4} H_{p p \pi}$, given in Fig. 3(d), we see new formations of gaps and bands, especially at regions close to $E_{\min }$ and $E_{\max }$. Near $\alpha=2$ and $E \simeq \pm 4$, there is a formation of new gaps. In addition, the bands in the region $1 \leqslant E \leqslant 3 \mathrm{eV}$ (and also the symmetric region with respect to the $E=0 \mathrm{eV}$ line) and $\alpha$ between 3 and 4 start forming separate groups, and these groups have tendencies to approach the $E=0 \mathrm{eV}$ line. Since the spectrum is periodic, the same structure can be observed for the values of $\alpha$ with a period of 4 . As we reduce the hopping constant of atom $E$ to one half of the usual tight-binding parameter, we observe that those groups form distinct "bat" -shaped regions within a large gap, as seen in Fig. 3(e). We see similar behavior for the bands at around $E= \pm 5 \mathrm{eV}$. For those regions, there also new gap formations, and the bands start to group. We continue to reduce $H_{p p \pi}^{E}$ to the one fourth and one fifth of $H_{p р л}$, and the corresponding spectra are given in Figs. 3(f) and $3(\mathrm{~g})$, respectively. For those graphs, we observe that the central bands lying around the $E=0 \mathrm{eV}$ line with $\alpha$ between 1 and 4 have shrunk, and the bat-shaped group starts to merge to $E=0 \mathrm{eV}$. We also observe that the central gaps are in approximately triangular shapes. The same sharpening of the gap boundaries can be observed for other large gaps. When we go to the limiting case, where $H_{p p \pi}^{E}=0$, given by Fig. 3(h), we obtain the vacancy case. ${ }^{19}$ For this case, the boundaries of the central and large gaps are sharpened, and they gain a more triangular shape. Also the shrunken bands and the bat-shaped groups have now collapsed to the $E=0 \mathrm{eV}$ line. So we see that the new formations of gaps and bands are due to impurity atoms. As the hopping constant of this impurity atom is reduced to smaller values, these new bands are decoupled from 
the rest of the system. In addition the impurity atom reduces the scale of energy. We also remark that these states due to the point defects (impurity or vacancy) are highly localized on the defect atoms which are not expected to contribute to the conduction. The other limit for the impurities is the case where the impurity atom has a larger hopping constant than the rest of the atoms. We start with the impurity atom located at the position of atom $\mathrm{E}$ with stronger hopping constant $H_{p р \pi}^{E}=\frac{3}{2} H_{p р \pi}$, shown in Fig. 3(b). We see a formation of larger gaps at around $E_{\min }$ and $E_{\max }$, and bands within these regions are already grouped together. When we go one step further to Fig. 3(a), by fixing the hopping constant of the impurity to $H_{p p \pi}^{E}=2 H_{p p \pi}$, we come across the ribbon-like spectra located at the top and the bottom of the original spectrum. These ribbons have their own gaps and bands, and they are totally separated from the rest of the spectrum. Hence we can see that the lattice of impurity atoms with such a high hopping constant produces its own self-similar butterfly. Such a separate impurity band at the extrema of the spectrum would be expected to modify the magnetotransport properties of the system.

\section{B. First- and second-nearest neighbors}

In general, the second-nearest-neighbor interactions bring an extra envelope-like periodicity to the Hofstadter butterflies. ${ }^{22}$ By implementing the next-nearest-neighbor interactions into the calculations, we break the bipartite symmetry of the lattice; hence the spectrum is no longer symmetric around $E=0 \mathrm{eV}$. The Hofstadter butterflies with second-order interactions are presented in Fig. 4 with the same sequence of defect-atom hopping constants of Fig. 3. We observe that the general characteristics of the spectra are the same and concentrate on the differences. The gaps and bands are shifted with respect to each other, and the central $E=0 \mathrm{eV}$ line is broken due to the loss of bipartite symmetry. We see the same gaps and bands evolving as a function of impurity hopping constant strengths. So we can claim that the gaps and bands due to the impurity atom for the first-order interactions are steady against to addition of the second-order interactions. For the standard second-order hopping strengths as listed in Table I, major gaps in the spectrum remain open. Thus, conductance values in these major gaps should not be affected by the second-order hopping.

\section{Impurity states}

As the major qualitative features in the spectra calculated above are due to impurity states, we investigate the nature of these states in more detail. These impurity states appear near $E=0 \mathrm{eV}$ for $t^{\prime}<t\left(t^{\prime}=H_{p p \pi}^{E}\right.$ is the hopping strength of the impurity atom, and $t=H_{p p \pi}$ is the hopping parameter for the rest of the system) and at the extrema of the spectrum for $t^{\prime}>t$, as discussed. Thus we calculate the probability distribution in real space for two states: the one closest to $E=0 \mathrm{eV}$ and the one at the minimum energy. Our results are plotted in Figs. 5(a) and 5(b). For $t^{\prime}>t$ [Fig. 5(a)] we see that the impurity state is not strictly localized on the $E$ atom. In the limit of $t^{\prime} \longrightarrow \infty$ the probability to be on the $\mathrm{E}$ atom is 0.5 , and its nearest neighbors have a probability of $0.5 / 3$ each. We see that these limiting
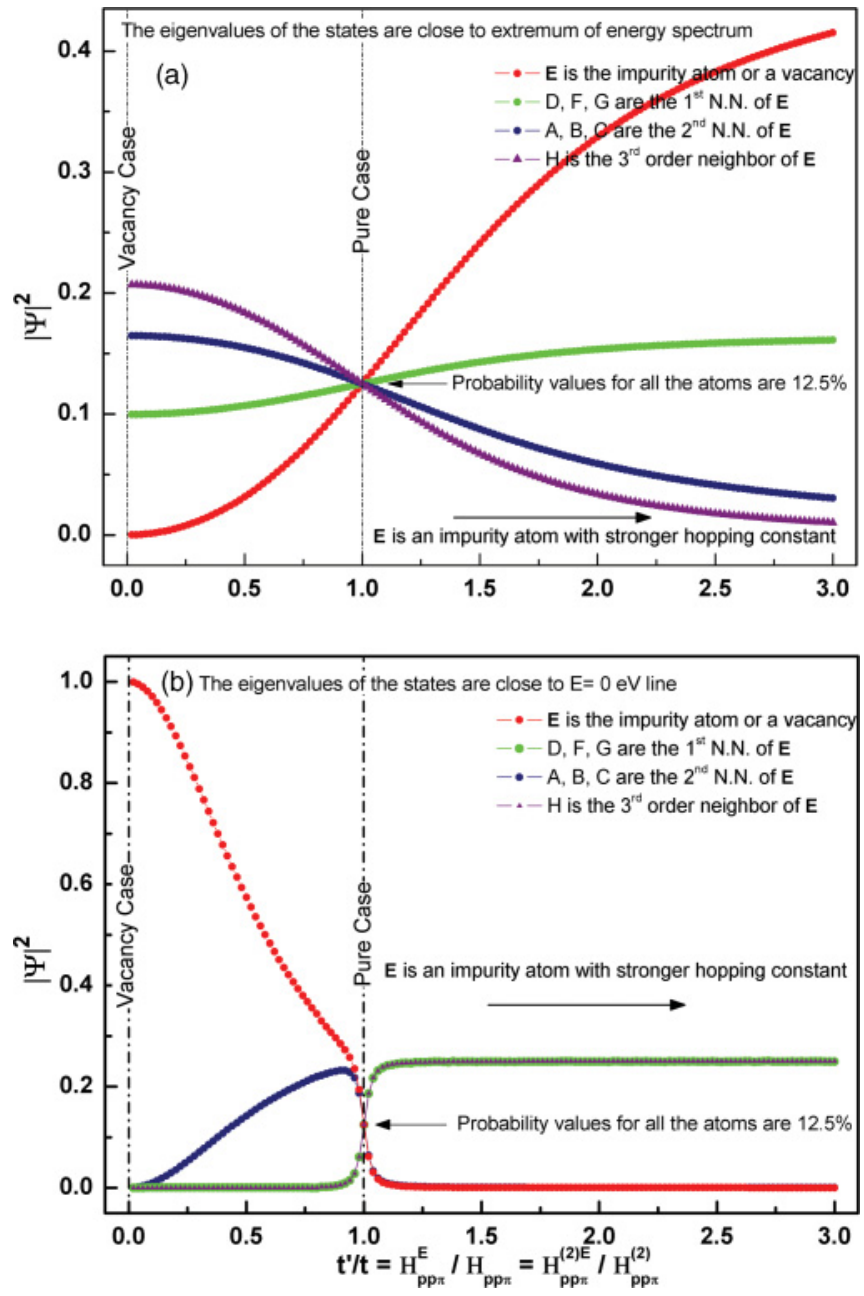

FIG. 5. (Color online) The values of $|\Psi|^{2}$ which give the projection of the probabilities of the wave functions onto the atomic positions are displayed as a function of impurity hopping strengths. The parameter are set to $p=1$ and $q=5, k_{x}=k_{y}=0$. The dash-dotted vertical lines display the special cases: vacancy and pure cases. (a) The eigenvalues for those eigenvectors are close to extremum of the energy spectrum. (b) The eigenvalues for those eigenvectors are close to the $E=0 \mathrm{eV}$ line. Note that there are several degenerate states near $0 \mathrm{eV}$, and we plot probabilities for only one of them.

values are asymptotically approached in Fig. 5(a). This state is separated in energy from the bulk of the spectrum forming ribbon-like structures observed in the Hofstadter butterflies, asymptotically approaching $\pm \sqrt{3} t^{\prime}$, as seen in Fig. 6. For $t^{\prime}<t$ [Fig. 5(b)] we observe rapid localization of the impurity state, validating our observation regarding the spectra around $E=0 \mathrm{eV}$. The vacancy case merits more discussion as the impurity atom is not only decoupled from the system but totally removed. Thus, in our calculation for the vacancy case we exclude the eigenvalue corresponding to this unphysical state from our spectra. However the absence of the atom modifies states which are not localized on the impurity but are close to $0 \mathrm{eV}$ in energy. In Fig. 3(h), all the bands are closed up on the $E=0 \mathrm{eV}$ line, in contrast to Fig. 4(h), where we observe a self-similar behavior of the energy spectrum near the 


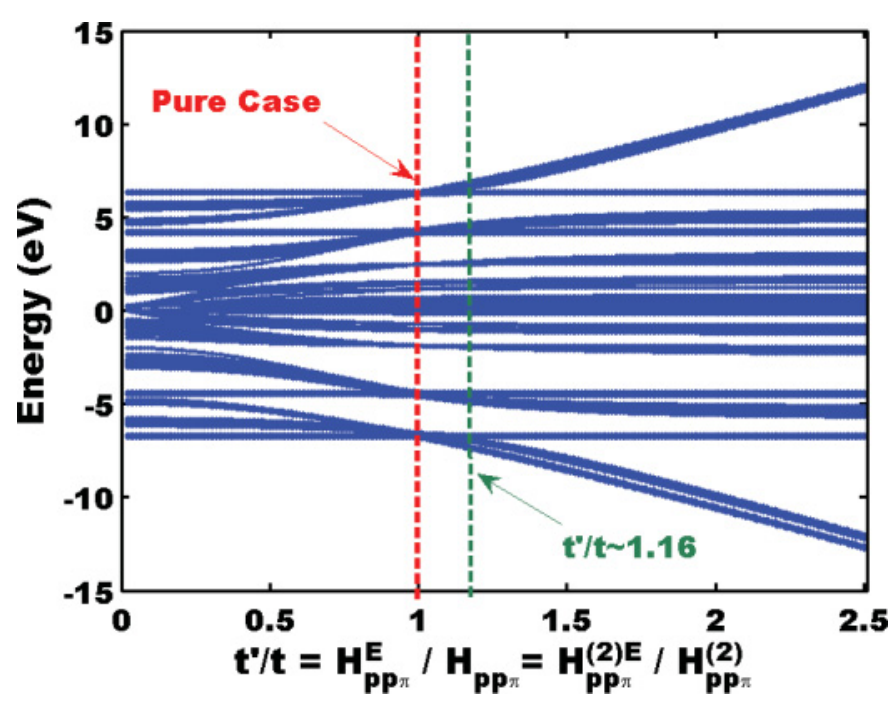

FIG. 6. (Color online) The change of band structure with respect to impurity hopping strength is displayed. The parameters are set to $p=78$ and $q=31, k_{x}=k_{y}=0$. The pure case is represented by the red dashed line, and the limiting value where the impurity bands start to leave the remaining spectrum is determined to be $t^{\prime} / t \simeq 1.16$.

$E=0 \mathrm{eV}$ line. It is important to emphasize that the states at 0 eV in Fig. 3(h) are not unphysical impurity states but collapsed bands. The reason for this collapse is that the interactions of the remaining atoms with the pseudoatom cancel each other exactly for eigenstates respecting bipartite symmetry for the nearest-neighbor case. For these states the breaking of the bipartite symmetry is especially important as their self-similar nature can only be observed when their energy can deviate from 0 [Fig. 4(h)].

It is important to understand the evolution of the impurity states as a function of the impurity hopping strengths and determine the critical values for $t^{\prime} / t$ at which major gaps open. We display the behavior of bands as a function impurity hopping strength in Fig. 6, including the next-nearest-neighbor hopping for a fixed value of the magnetic field. In Fig. 6, it is easy to discern states associated with the impurity atoms by their strong variation with the impurity hopping constant. We see that the width of the spectrum does not change significantly between the vacancy and pure case limits. We observe modifications in the bands and the creation of new gaps within this region. However, when going to the strong coupling limit, two bands from the top and the bottom of the energy leave the remaining spectrum and exhibit their own self-similar structures [corresponding to the ribbons observed in Figs. 3(a) and 4(a)]. The critical value for the separation is $H_{p p \pi}^{E} / H_{p p \pi}=H_{p p \pi}^{2(E)} / H_{p p \pi}^{2} \simeq 1.16$.

The reason for the self-similar structure within impurity bands and gaps is that we introduce the impurities to the system in a periodic way. In reality the impurities are scattered randomly over the entire sample. For such a sample we believe that these separated bands would still exist; however, the gaps and the bands would be blurred, and the bands would lose their self-similar structure due to the random distribution of the impurities.

\section{CONCLUSIONS}

We examined the effect of imperfections on the electronic structure of graphene in the presence of a perpendicular magnetic field. We used the tight-binding method with both the first- and the second-nearest-neighbor interactions included within an enlarged unit cell. We also showed the Hofstadter butterfly for pure graphene including all the orbitals. For the defect cases, the impurity atoms with smaller hopping constants result in highly localized states, and these states produce new gaps and bands in the energy spectra. As the hopping constant(s) of the impurity atom is reduced down to zero, we see that the bands are decoupled from the rest of the spectrum, and they merge at the symmetry line where $E=0$ $\mathrm{eV}$. So these kinds of impurities reduce the energy scale, and they mostly modify the spectrum around $E=0 \mathrm{eV}$. In contrast to this case, when the impurity atom has a larger hopping constant, the bands and gaps due to the impurity atom are totally separated from the original energy spectrum. These new gaps and bands produce their own self-similar collection of bands arranged into a ribbon. Such impurities also increase the overall energy scale. The second-nearest-neighbor interactions break the bipartite symmetry of the lattice and bring a new periodicity to the energy spectrum. The effects of impurity atoms on the energy spectrum are the same in the sense of new gaps and bands except for small shifts when we consider the next-nearest-neighbor hopping.

\section{ACKNOWLEDGMENTS}

M.O.O. acknowledges support from TÜBITAK, the Scientific and Technological Research Council of Turkey (Grant No. TBAG 109T267). O.G. acknowledges the support of the Turkish Academy of Sciences (TÜBA).
${ }^{1}$ P. R. Wallace, Phys. Rev. 71, 622 (1947).

${ }^{2}$ K. S. Novoselov, A. K. Geim, S. V. Morozov, D. Jiang, Y. Zhang, S. V. Dubonos, I. V. Grigorieva, and A. A. Firsov, Science 306, 666 (2004).

${ }^{3}$ K. S. Novoselov, D. Jiang, F. Schedin, T. J. Booth, V. V. Khotkevic, S. V. Morozov, and A. K. Geim, Proc. Natl. Acad. Sci. USA 102, 10451 (2005).

${ }^{4}$ A. H. Castro Neto, F. Guniea, N. M. R. Peres, K. S. Novoselov, and A. K. Geim, Rev. Mod. Phys. 81, 109 (2009).
${ }^{5}$ R. Peierls, Z. Phys. 80, 763 (1933).

${ }^{6}$ D. R. Hofstadter, Phys. Rev. B 14, 2239 (1976).

${ }^{7}$ C. Albrecht, J. H. Smet, K. von Klitzing, D. Weiss, V. Umansky, and H. Schweizer, Phys. Rev. Lett. 86, 147 (2001).

${ }^{8}$ J. G. Analytis, S. J. Blundell, and A. Ardavan, Am. J. Phys. 72, 613 (2004).

${ }^{9}$ Y. Hasegawa, Y. Hatsugai, M. Kohmoto, and G. Montambaux, Phys. Rev. B 41, 9174 (1990).

${ }^{10}$ J.-M. Hou and W.-X. Yang, Phys. Lett. A 373, 2774 (2009). 
${ }^{11}$ Y. Hasegawa and M. Kohmoto, Phys. Rev. B 74, 155415 (2006).

${ }^{12}$ R. Rammal, J. Phys. (Paris) 46, 1345 (1985).

${ }^{13}$ G. Gumbs and P. Fekete, Phys. Rev. B 56, 3787 (1997).

${ }^{14}$ N. Nemec and G. Cuniberti, Phys. Rev. B 75, 201404 (2007).

${ }^{15}$ Z. Wang, F. Liu, and M. Y. Chou, Bull. Am. Phys. Soc. 56 (2011), http://meetings.aps.org/link/BAPS.2011.MAR.X36.3.

${ }^{16}$ R. Bistritzer and A. H. MacDonald, Phys. Rev. B 84, 035440 (2011).
${ }^{17}$ P. Moon and M. Koshino, Phys. Rev. B 85, 195458 (2012).

${ }^{18}$ N. Nemec and G. Cuniberti, Phys. Rev. B 74, 165411 (2006).

${ }^{19}$ A. L. C. Pereira and P. A. Schulz, Phys. Rev. B 78, 125402 (2008).

${ }^{20}$ P. G. Harper, Proc. Phys. Soc. London, Sect. A 68, 874 (1955).

${ }^{21}$ J.-C. Charlier, Ph. Lambin, and T. W. Ebbesen, Phys. Rev. B 54, 8377 (1996).

${ }^{22}$ Y. Hatsugai and M. Kohmoto, Phys. Rev. B 42, 8282 (1990). 